\title{
High contrast imaging with the JWST NIRCAM coronagraph
}

Joseph J. Green, Charles Beichman, Scott A. Basinger, Scott Horner, Michael Meyer, et al.

Joseph J. Green, Charles Beichman, Scott A. Basinger, Scott Horner, Michael Meyer, David C. Redding, Marcia Rieke, John T. Trauger, "High contrast imaging with the JWST NIRCAM coronagraph," Proc. SPIE 5905, Techniques and Instrumentation for Detection of Exoplanets II, 59050L (14 September 2005); doi: 10.1117/12.619343

SPIE. Event: Optics and Photonics 2005, 2005, San Diego, California, United States 


\title{
High Contrast Imaging with the JWST NIRCAM Coronagraph
}

\author{
Joseph J. Green ${ }^{\mathrm{a}}$, Charles Beichman ${ }^{\mathrm{b}}$, Scott A. Basinger ${ }^{\mathrm{a}}$, Scott Horner ${ }^{\mathrm{d}}$, Michael Meyer ${ }^{\mathrm{c}}$, \\ David C. Redding ${ }^{\mathrm{a}}$, Marcia Rieke ${ }^{\mathrm{c}}$, and John T. Trauger ${ }^{\mathrm{a}}$ \\ a Jet Propulsion Laboratory, California Institute of Technology, Pasadena California 91109 \\ ${ }^{\boldsymbol{b}}$ Michelson Science Center, California Institute of Technology, Pasadena California 91125 \\ ${ }^{c}$ Steward Observatory, University of Arizona, Tucson, AZ 85721 \\ ${ }^{d}$ Lockheed Martin Adv. Tech. Center, Palo Alto, CA 94304
}

\begin{abstract}
Relative to ground-based telescopes, the James Webb Space Telescope (JWST) will have a substantial sensitivity advantage in the $2.2-5 \mu \mathrm{m}$ wavelength range where brown dwarfs and hot Jupiters are thought to have significant brightness enhancements. To facilitate high contrast imaging within this band, the Near-Infrared Camera (NIRCAM) will employ a Lyot coronagraph with an array of band-limited image-plane occulting spots. In this paper, we provide the science motivation for high contrast imaging with NIRCAM, comparing its expected performance to that of the Keck, Gemini and $30 \mathrm{~m}$ (TMT) telescopes equipped with Adaptive Optics systems of different capabilities. We then describe our design for the NIRCAM coronagraph that enables imaging over the entire sensitivity range of the instrument while providing significant operational flexibility. We describe the various design tradeoffs that were made in consideration of alignment and aberration sensitivities and present contrast performance in the presence of JWST's expected optical aberrations. Finally we show an example of a two-color image subtraction that can provide $10^{-5}$ companion sensitivity at sub-arcsecond separations.
\end{abstract}

Keywords: Coronagraph, high contrast imaging, extrasolar planets, protoplanetary disks, NIRCAM, JWST

\section{INTRODUCTION}

NIRCAM, one of the primary science instruments for the James Webb Space Telescope (JWST), will conduct imaging surveys in broad, intermediate, and narrow-band filters in order to attack a wide range of problems from detecting first light objects in the early universe to questions concerning the origin and evolution of planetary systems ${ }^{1}$. An exciting application of NIRCAM will be the search for planetary companions orbiting a variety of stars as well as the study of gas and dust disks from which planets form. This paper addresses the observations possible with the NIRCAM coronagraph in two ways. In the first part of the paper, we use a simple parameterized model of coronagraphic performance to compare JWST with different ground-based telescopes to understand JWST's unique discovery space. This analysis shows that JWST is uniquely suited to find and characterize planetary mass companions orbiting stars beyond $0.5 \sim 1$ " in the 3-5 $\mu \mathrm{m}$ region. Potential targets include the nearest young $\mathrm{M}, \mathrm{L}$ and $\mathrm{T}$ stars, a few nearby stars with planets known from radial velocity studies, and the nearest young stars. With JWST we can take full advantage of the fact that Jupiter-sized bodies emit strongly in the $4.8 \mu \mathrm{m}$ region ${ }^{2,3}$ to find low mass objects inaccessible at shorter wavelengths. Secondly, the JWST point spread function will be extremely stable on the timescale of hours compared to ground based telescopes equipped with adaptive optics. Thus, even at 1-3 $\mu \mathrm{m}$, the NIRCAM coronagraph will be well suited for the study of very faint, diffuse structures such as protoplanetary and debris disks around bright stars. The second part of this paper describes a detailed model of the JWST coronagraph based on a complete diffraction analysis of the JWST optical system. This model will be used by the NIRCAM team to make accurate performance predictions and to assess tradeoffs in the design of NIRCAM as the instrument and telescope evolve.

\footnotetext{
* Contact author information:

E-mail: Joseph.J.Green@jpl.nasa.gov, 4800 Oak Grove Dr., M/S 306-336, Pasadena CA 91109.
} 


\section{SCIENCE GOALS}

\subsection{Search for Planetary Companions}

Over the next 20 years searches for planetary companions will eventually become sensitive to Earth mass planets in the habitable zone $(\sim 1 \mathrm{AU})$ using the Terrestrial Planet Finder. But today, the state of the art in direct detection has resulted in the identification of a warm gas giant planet in a wide orbit around a nearby young $(\sim 10 \mathrm{Myr})$ brown dwarf ${ }^{4}$. JWST represents an important intermediate step in this evolving set of capabilities. As described in this paper, JWST will be able to search for Jupiter-sized planets in the following nearby systems:

- At a wavelength of $4.8 \mu \mathrm{m}$, self-luminous Jupiters (1-5 $\left.\mathrm{M}_{\mathrm{Jup}}\right)$ orbiting $\mathrm{M}$ stars and brown dwarfs as old as $5 \mathrm{Gyr}$ will be detectable at $>5 \mathrm{AU}$ in perhaps two dozen systems. In particular, observations at $4.8 \mu \mathrm{m}$ will be important where Jovian planets are thought to be very bright relative to shorter wavelengths ${ }^{2,3}$. These objects are likely to be undetectable at shorter wavelengths using ground-based telescopes.

- Self-luminous Jupiters ( $\left.>1 \mathrm{M}_{\mathrm{Jup}}\right)$ with ages less than $100 \mathrm{Myr}$ will be observable at orbital radii beyond $25 \mathrm{AU}$ $(\sim 1 "$ at $25 \mathrm{pc})$ around a few hundred young stars within $25-150 \mathrm{pc}$ at wavelengths longward of $3 \mu \mathrm{m}$. Groundbased observations using adaptive optics have already found such objects at 1-2 $\mu \mathrm{m}$ and will doubtless find more in the coming years (e.g. Chauvin et al $2005^{4}$ ). However, JWST can provide important information in the 3-5 $\mu \mathrm{m}$ region for known objects using a variety of medium and narrow band filters, as well as finding older, cooler objects invisible at $1-2 \mu \mathrm{m}$.

- While Jupiters confirmed to exist from radial velocity studies would probably NOT be detectable by JWST ${ }^{5}$, planets at greater orbital separation around the most favorable radial-velocity (RV) might be detectable (e.g. $\varepsilon$ Eri, $55 \mathrm{Cnc}, 47 \mathrm{UMa}$ ). The existence of such planets is often suspected from as yet incomplete RV datasets.

\subsection{The Search for Protoplanetary and Debris Disks}

The study of jets and outflows from young stars guides our understanding of the star formation process as well as the assembly of disks that are a necessary prerequisite to planet formation. The study of rings, gaps, and asymmetric structures in disks surrounding mature stars can yield tantalizing clues to the evolution of planetary systems (e.g. Kalas et. al. 2005, [6], and Ardila et. al. (2005), [7]). For these studies we need to block the light from the central star to reveal primordial disks (arising due to the conservation of angular momentum during the star formation process) or debris disks (dust generated through the collisions of planetesimals). Primordial disks can be quite bright relative to the parent star, but we wish to study them in narrow spectral bands ( 1\% filters) to isolate atomic and molecular emission lines. Debris disks are very faint compared to the central star so that the ability to reject starlight at extreme levels is essential to see disks even 100-1000 times brighter than our own zodiacal cloud. Observations of these disks (seen in reflected light) in moderate passbands $(10 \%)$ will enable us to study the morphology of disks and to look for broad mineralogical features. The great stability of the JWST operating above the atmosphere will allow us to remove residual starlight to reveal faint diffuse structures around nearby stars.

Recent surveys with the Spitzer Space Telescope have revealed dozens of debris disks. Models of the spectral energy distributions assembled from Spitzer data are often degenerate with multiple solutions capable of explaining the observations. Small particles achieve the same equilibrium temperature as larger particles at larger distances from the star, leading to vastly different physical conditions and inferred planetary configurations. For example, $0.5 \mu \mathrm{m}$ grains will achieve a temperature of $40 \mathrm{~K}$ at a distance $>300 \mathrm{AU}$ from the central star while $50 \mu \mathrm{m}$ grains will reach the same temperature at $<50 \mathrm{AU}$. A high performance coronagraph on JWST operating at wavelengths from 0.8-2.3 $\mu \mathrm{m}$ will enable us to resolve these disks, breaking such model degeneracies and possibly revealing asymmetries in scattered light that could be the tell-tale signs of planets (e.g. Kalas et al. 2005, [6]). Narrow-band spectroscopic observations will enable the assessment of the composition of the dust in these disks and contribute to our understanding of how volatiles such as water and organic material are transported in planetary systems through scattering of planetesimals (e.g. Emery $\&$ Brown 2003, [8]). Such processes are thought to have occurred early in our solar system's history and represent one piece of the puzzle concerning how life arose on our planet. 
Table 1. Telescope parameters for science modeling.

\begin{tabular}{rrrrr}
\hline \multirow{2}{*}{ Parameter } & \multicolumn{4}{c}{ Telescope } \\
& JWST & Keck & Gemini-ExAO & TMT \\
\hline Diameter (m) & 6 & 10 & 8 & 30 \\
Segment (m) & 1.00 & 1.80 & 8.00 & 1.50 \\
Overall Efficiency & 0.50 & 0.25 & 0.25 & 0.25 \\
Emissivity & 0.10 & 0.30 & 0.20 & 0.30 \\
Temperature (K) & 45 & 290 & 290 & 290 \\
Wave Front Error (nm) & 130 & 300 & 50 & 250 \\
Strehl Ratio (2.2 mm) & 0.871 & 0.480 & 0.980 & 0.601 \\
Strehl Ratio (4.8 mm) & 0.971 & 0.857 & 0.996 & 0.898 \\
Seeing (", FWHM) & 0.000 & 0.250 & 0.250 & 0.250 \\
Airglow (<2.2 mm, MJy/sr) & 0 & 100 & 100 & 100 \\
Limiting Q (planet/halo)* & 0.100 & 0.500 & 0.100 & 0.500 \\
\hline
\end{tabular}

*Increase ground based telescopes by $\mathrm{x} 5$ for diffuse emission

\subsection{Parameterized Performance Model}

By its launch in 2012, JWST will be neither the largest telescope nor have the lowest level of uncorrected wavefront error. Eight to ten meter ground-based telescopes such as Keck, Gemini, or the VLT will be in operation, some with extreme Adaptive Optics systems capable of a high degree of correction in a small field around a bright star. There may even be a $30 \mathrm{~m}$ telescope (TMT) with a very small diffraction limited beam. While the ground based telescopes suffer from high thermal or $\mathrm{OH}$ backgrounds that greatly reduce their sensitivity relative to a space-based system, the noise floor in a coronagraph, particularly close to the central star, can be set by diffracted or scattered starlight, not large-scale backgrounds. Thus it is important to develop a simple model that takes these different effects into account in comparing different telescopes.

Table 1 lists the characteristics of the four idealized telescopes we consider in this analysis: JWST, Keck with its existing Adaptive Optics (AO), Gemini with an illustrative Extreme AO system, and a $30 \mathrm{~m}$ telescope with modest AO. To calculate the background noise we first evaluate the uniform background from thermal emission and/or airglow. To this we add the diffracted and scattered starlight, which we assume follows a core-halo distribution outside the inner working angle $(I W A=4 \lambda / D$ at a wavelength $\lambda$ and a telescope of diameter $D)$. Thus, the surface brightness, $I(R)$, in the image plane at a distance $R$ from the star (outside the IWA) is given by

$$
I(R) \propto \text { Thermal }+ \text { Airglow }+ \text { Strehl } \cdot a \cdot\left(\frac{R}{I W A}\right)^{-3}+(1-\text { Strehl }) \cdot \exp \left(-\left(1.665 \cdot \frac{R}{F W H M}\right)^{2}\right),
$$

where $a$ is a constant derived from considering the diffracted energy not encircled within the IWA. For the groundbased telescopes the scattered starlight, (1-Strehl) of the total, follows a Gaussian distribution with a 0.25" FHWM. For JWST with no atmosphere, we assume that the residual (1-Strehl) scattered light falls in a Gaussian halo with a size given by the diffraction from a segment, $\mathrm{FHWM}=\lambda D_{\text {segment. }}$ We ignore the effects of diffraction from the segments in this idealized analysis; these effects are included in the detailed modeling of JWST presented in the next section. A perfect coronagraph would, of course, completely remove the stellar terms in Eqn. (1).

To take into account the limited performance of the coronagraph due to residual wavefront errors and aberrations, we assume that the coronagraph attenuates the starlight terms in Equation (1) by a factor of (1-Strehl). Thus, the better the wavefront error, the better the Strehl and the lower the effects of residual starlight. Figures 1a,b show the backgrounds in the focal planes for four telescopes at 1.25 and $4.8 \mu \mathrm{m}$ assuming that either a 5 or 10 mag star is being observed. At $1.25 \mu \mathrm{m}$ the $5 \mathrm{mag}$ star dominates the background for the ground-based telescopes out to $\mathrm{R}=1-1.5$ " when the airglow limit is reached. For JWST the 5 or 10 mag star dominates the zodiacal scattered light well beyond this radius. At $4.8 \mu \mathrm{m}$, the ground-based thermal background dominates the stellar background at all radii, but for JWST the residual stellar background from a $5 \mathrm{mag}$ star dominates the zodiacal emission well beyond 1.5". Residual starlight is an important noise source for JWST and reduces the advantages of its low thermal background close to a bright star. 

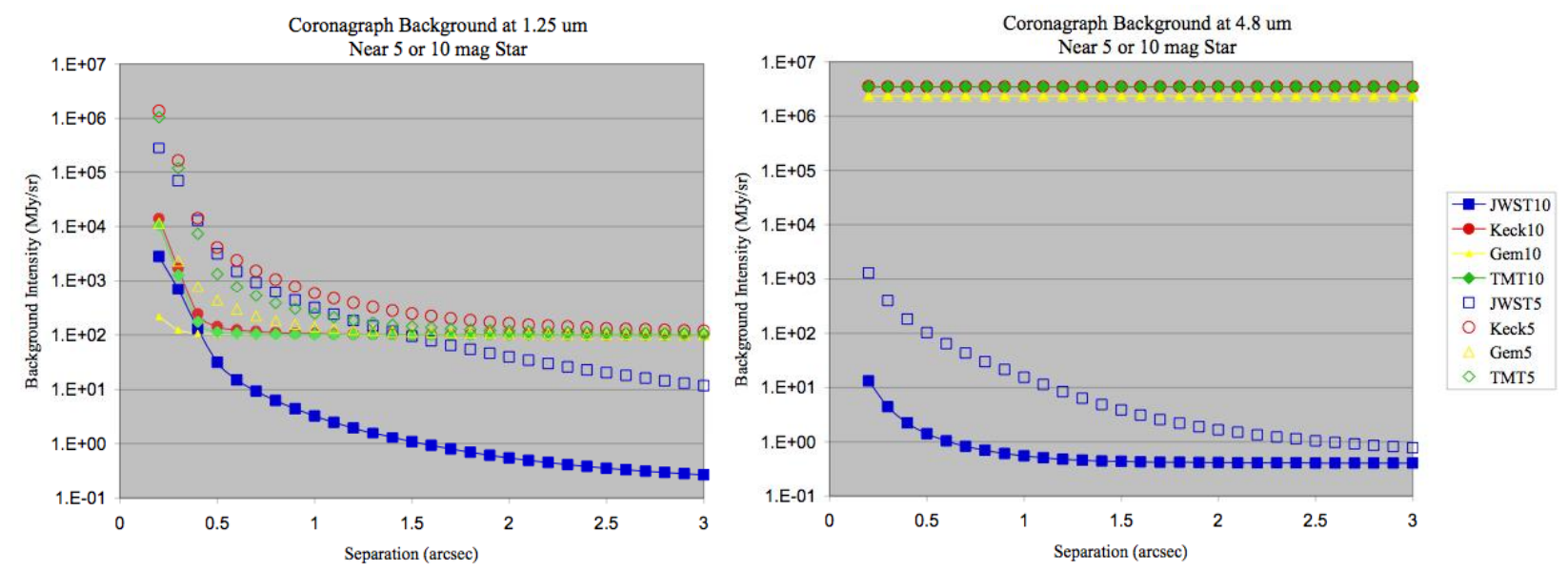

Figure 1. Residual backgrounds in the focal plane due to scattered and diffracted starlight, telescope and atmospheric backgrounds (if any). Curves are shown for a central star of 5 (open symbols) or 10 mag (solid lines and symbols) as a function of separation from the star and for four telescopes as described in Table 1: JWST (squares), Keck (circles), Gemini with extreme adaptive optics (triangles), and $30 \mathrm{~m}$ TMT (diamonds).

To calculate the sensitivity limit for point sources we integrate for a time, $\tau$, within a diffraction-limited beam of $\mathrm{A} \Omega=\lambda^{2}$ and a total optical efficiency $\varepsilon$. In the simplest case, the noise would be given by the square root of the number of collected noise electrons so that additional integration time could ameliorate the effects of increased background. More realistically, however, wavefront errors produce residual "speckles" in the image that result in a corrugated background of faint false sources against which one must find a true, faint point source. The brightness of these speckles is approximated by the residual stellar surface brightness at a position $R, I(R)$ in an $A \Omega=\lambda^{2}$ beam, that produces a noise floor that does not improve with integration time. Because the speckles are a fixed pattern noise, various techniques can reduce, but never eliminate, this noise source, we introduce the parameter $Q$ which describes how far below the residual speckle brightness we can still find sources. We adopt $Q=0.1$ for JWST and an extreme AO system on Gemini on the assumption that the stable environment of space or a high speed AO system with scintillation correction will allow these telescopes to reach well below the residual stellar halo. For Keck and TMT we adopt $Q=0.5$ which is close to present day values". For the study of protoplanetary and debris disks the critical parameter is the limiting surface brightness which has, to date, proven to be a much more challenging observational problem for groundbased than space-based telescopes due to rapidly time variable atmospheric effects. To account for the ground-space differences in calibrating images to look for diffuse emission, we increase the $\mathrm{Q}$ factor in the model calculations by a factor of 5 for the ground-based telescopes.

\subsection{Model Results}

Figures 2a-d summarize the limiting performance for planet detection at a signal to noise ratio (SNR) of 10 in $\tau=10^{4} \mathrm{sec}$ and a $25 \%$ spectral bandpass with the four telescopes for a planet orbiting a 5 or 10 mag star at four infrared wavelengths, $1.25,2.2,3.4$, and $4.8 \mu \mathrm{m}$ and for separations out to $3 "$. The curves show that at short wavelengths, 1.25 and $2.2 \mu \mathrm{m}$, JWST is at a definite disadvantage relative to either any of the ground-based telescope within 1.5" of either a 5 or 10 mag parent star, but especially when compared to an $8 \mathrm{~m}$ telescope with extreme AO. At $3.5 \mu \mathrm{m}$ an extreme AO system has a slight advantage out to 1 " for a 5 mag parent star at which point the low intrinsic background of JWST takes over and gives the space-based telescope a great sensitivity advantage. For a $10^{\text {th }}$ mag parent star, the JWST coronagraph has an immediate sensitivity advantage at all separations. Finally, at $4.8 \mu \mathrm{m}$, JWST has a enormous sensitivity advantage relative to the ground-based telescopes for all separations and parent star magnitudes; only the 30 m TMT system comes close in performance within the central 1". 

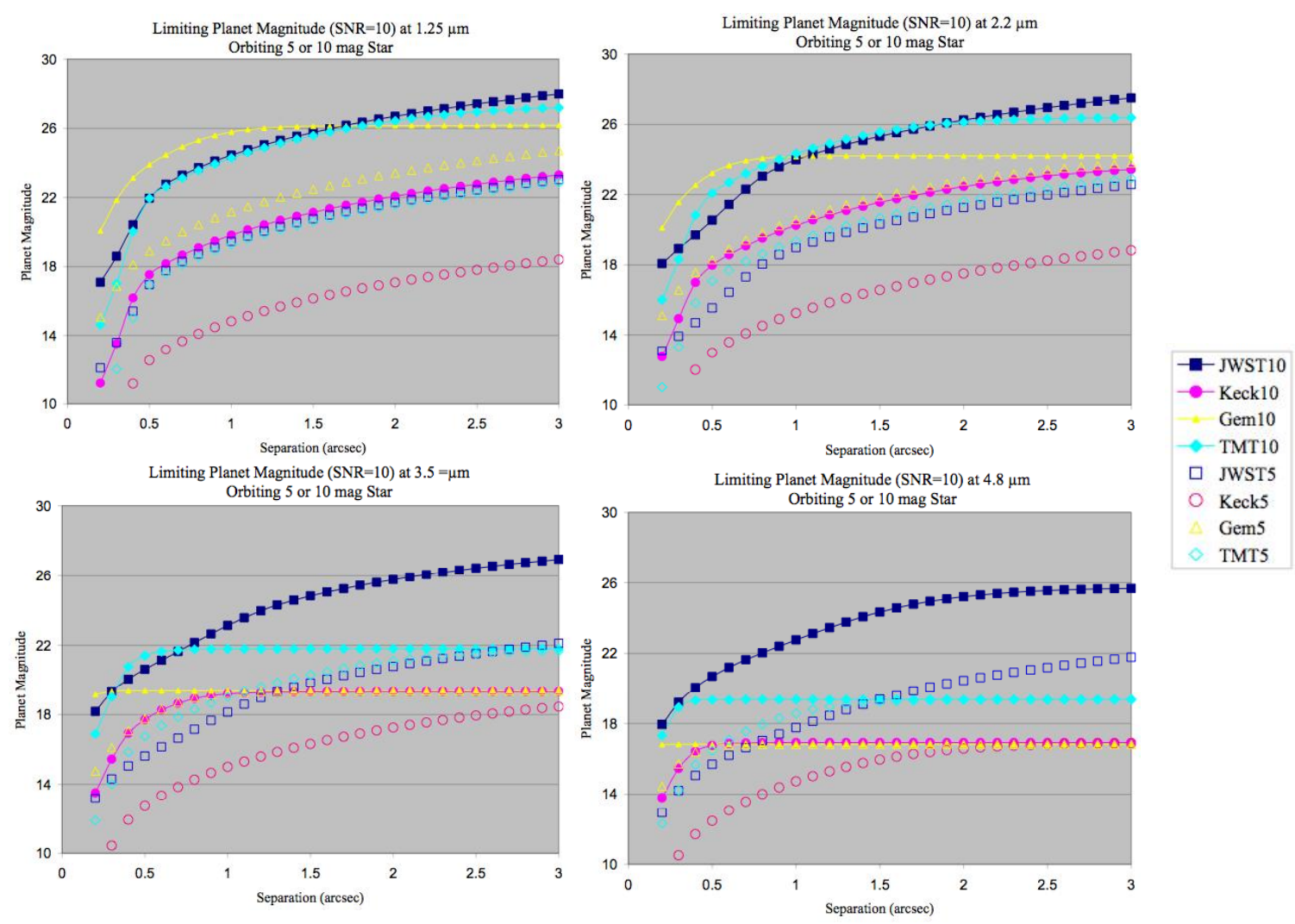

Figure 2. . Limiting companion magnitude for signal to noise ratio (SNR) of 10 in $10^{4} \mathrm{sec}$ for a planet orbiting a star of brightness $=5$ (open symbols) or $10 \mathrm{mag}$ (solid lines and symbols) as a function of separation. Curves are shown for four telescopes as described in Table 1: JWST (squares), Keck (circles), Gemini with extreme adaptive optics (triangles), and $30 \mathrm{~m}$ TMT (diamonds).

Figure 3 compares the performance of the four telescopes for the study of debris and planetary disks. Reaching a limiting surface brightness of 100-1,000 times the brightness of our own zodiacal cloud (a few tenths of a MJy/sr) is possible within 1-3" of a 5 mag star. Much lower levels would be achievable around a fainter T Tauri star like TW $\mathrm{Hya}^{10}$. In this case only Gemini using extreme Adaptive Optics performs comparably to JWST in the $1-2 \mu \mathrm{m}$ region within 1-2". At greater separations and at longer wavelengths, JWST has important advantages in looking for disks due to the stability of its residual scattered light and low backgrounds. Finally, we note that the use of an optimized coronagraph in an unaberrated beam and the stability of a telescope in an L2 orbit should give JWST significant advantages relative to HST in looking for debris disks ${ }^{5}$.

In summary, the performance models suggest that although at 1.25 and $2.2 \mu \mathrm{m}$ large ground based telescopes equipped with extreme Adaptive Optics will be superior for finding faint companions within 3" of bright parent stars, JWST will be a powerful competitor for fainter target stars, i.e. young $\mathrm{T}$ Tauri stars, or at distances beyond $\sim 3$ ". At wavelengths longer than $2.2 \mu \mathrm{m}$, the natural advantages of a cold telescope above the atmosphere quickly reassert themselves making JWST the premier facility for finding faint companions close to bright stars. The stability of the residual starlight in the NIRCAM coronagraph will make it possible to identify faint structures in protoplanetary and debris disks. 

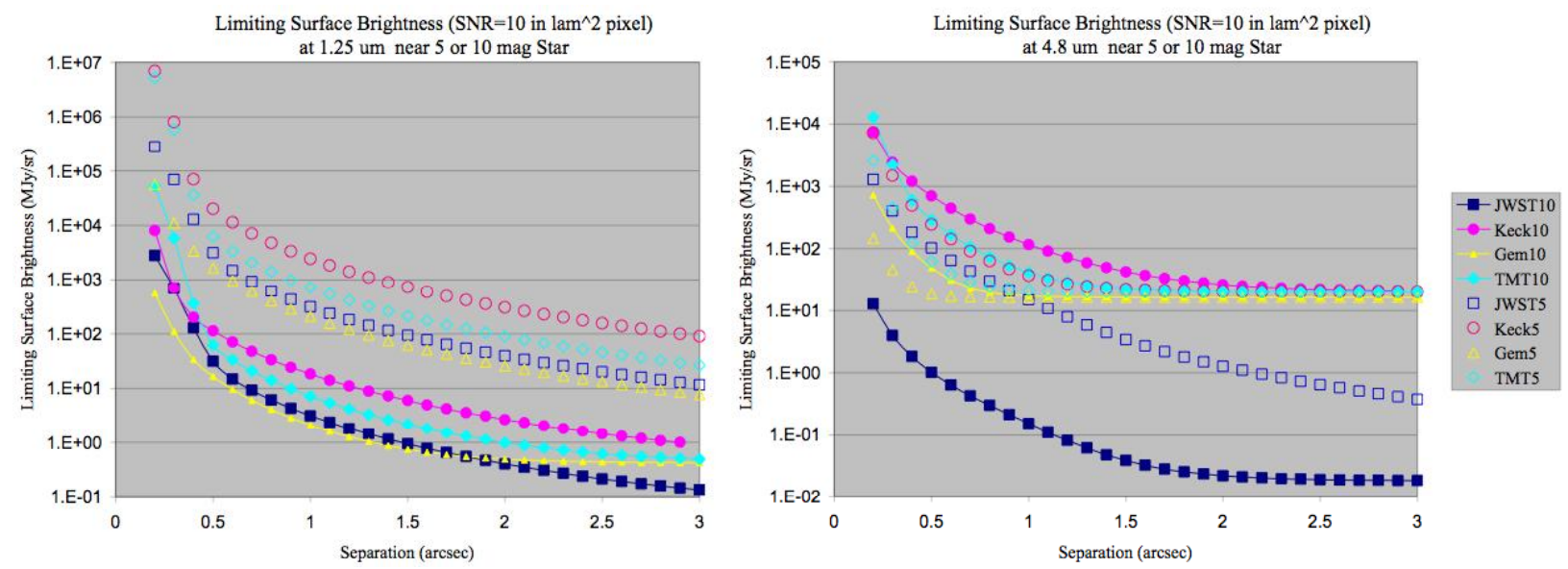

Figure 3. Limiting surface brightness for signal to noise ratio (SNR) of 10 in $10^{4} \mathrm{sec}$ for diffuse emission near a star of brightness $=$ 5 (open symbols) or $10 \mathrm{mag}$ (solid lines and symbols) as a function of separation. Curves are shown for four telescopes as described in Table 1: JWST (squares), Keck (circles), Gemini with extreme adaptive optics (triangles), and $30 \mathrm{~m}$ TMT (diamonds).

\section{NIRCAM CORONAGRAPH DESIGN}

\subsection{NIRCAM Accomodations for Coronagraphic Elements}

The architecture NIRCAM is well suited to the implementation of a Lyot coronagraph for high contrast imaging. . Lyot coronagraphs use a combination of a focal plane occulter and a downstream pupil stop to suppress stellar diffracted light. If the occulter is apodized such that it forms a band-limited function, then the stellar diffracted light can be perfectly eliminated ${ }^{11}$.

At the focus after the NIRCAM pickoff mirror, there is place for an array of 5 occulting spots, each one providing a 20 " 20 " field of view (FOV). A filter wheel at a subsequent image of the pupil has slots for two Lyot stops. Although the plate containing the occulting spot array is outside the nominal NIRCAM FOV, a wedged-glass plate co-located with each Lyot stop offsets the focal-plane FOV to co-align with the occulting spots.. While there is a single occulting spot array for the entire NIRCAM instrument, there are two Lyot stop slots available for both the long- and short-wave arms in each of the two cameras.

\subsection{Coronagraph Designs}

As motivated by our science analysis, there is desire to image both faint $\left(\sim 10^{-5}\right)$ companion sources and extended objects and at sub-arcsecond inner working angles (IWA). Competing with these objectives are the realities that JWST presents to the instrument. With the diffracted light from a $6.5 \mathrm{~m}$ segmented aperture, one has to compromise between the IWA at the longest wavelength of interest with the throughput of subsequent Lyot stop design.

In Fig. 4, we show the array of occulting spots suitable for NIRCAM high contrast imaging. With each spot we show the limiting imaging wavelength and identify which Lyot stop is compatible. In our array, we selected 3 radialsombrero $^{2}$ occulting spots that have intensity transmission of the form

$$
T(x, y)=\left(1-\left(\frac{2 J_{1}\left(\frac{\pi}{\sigma} \sqrt{x^{2}+y^{2}}\right)}{\frac{\pi}{\sigma} \sqrt{x^{2}+y^{2}}}\right)^{2}\right)^{2} .
$$



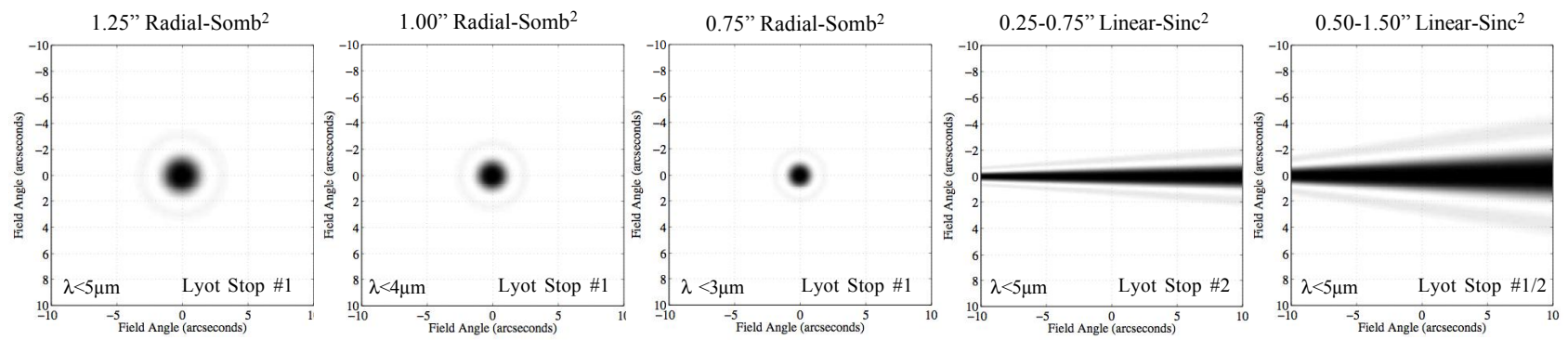

Figure 4. The NIRCAM occulting spot designs are shown along with the optical-filter compatibility range and Lyot stop requirement. The Lyot stop designs are shown in Fig. 5.

The size of occulting spot ultimately determines the IWA. While we would like to make this as tight as possible, the resulting efficiency in the Lyot stop design becomes worse for smaller occulters at the same imaging wavelength. In addition to blocking the residual diffracted stellar light, the Lyot stop must be desensitized to potential misalignments of the nominal pupil image. As can be seen in Fig. 5, this desensitization has the consequence of greatly reducing the Lyot stop efficiency. Without concern for pupil alignment the Lyot stop may let $\sim 50 \%$ of the light through. With the complex edge-structure of the JWST pupil (segments + spiders), the Lyot stop must be narrowed to admit only $20 \%$ of the companion light through. While a potential companion PSF will be less compact and fainter, the stricter Lyot stop designs will help guarantee high contrast imaging performance with immunity to pupil image misalignment and large error from segment edge roll-off.

A consequence of the desensitized Lyot stops is that it drives the design to favor larger occulting spots. The combination enables reasonable net coronagraph efficiency at the cost of IWA. Given these trades, we found with the F480M filter $(\lambda=4.8 \mu \mathrm{m} \pm 5 \%)$, the tightest practical IWA was about $1.25 \%$. This is where the occulter transmission reaches $50 \%$. While all other filters will work with this design, we also provide 1.00 " and 0.75 " occulting spots to facilitate smaller IWA while using filters at shorter wavelengths. These small spots work with shorter wavelength because the residual diffracted light at the Lyot pupil is dictated in part by the ratio of the spot size to the point-spread function (PSF) core size.

In addition to these three spots, we have specified two linear-sinc ${ }^{2}$ occulters with intensity transmission of the form

$$
T(x, y)=\left(1-\left(\frac{\sin \left(\frac{\pi}{\sigma} \cdot \frac{y}{x-x_{o}}\right)}{\frac{\pi}{\sigma} \cdot \frac{y}{x-x_{o}}}\right)^{2}\right)^{2} .
$$

As can be seen in Fig. 4, these occulters have a spatially variant width. This adds a lot of operational flexibility to tune the coronagraph to optimize the IWA for any filter. In Fig. 6, we show the nominal pointing objectives that provided the tightest IWA for each filter in the long-wave arm of NIRCAM. If the pupil image alignment error exceeds the expected $\sim 2.5 \%$, these pointing objectives can be shifted towards the wider end of the occulter. Likewise, if the pupil alignment is better than expect, the pointing objectives can be shifted to tighten the IWA as much as possible. 

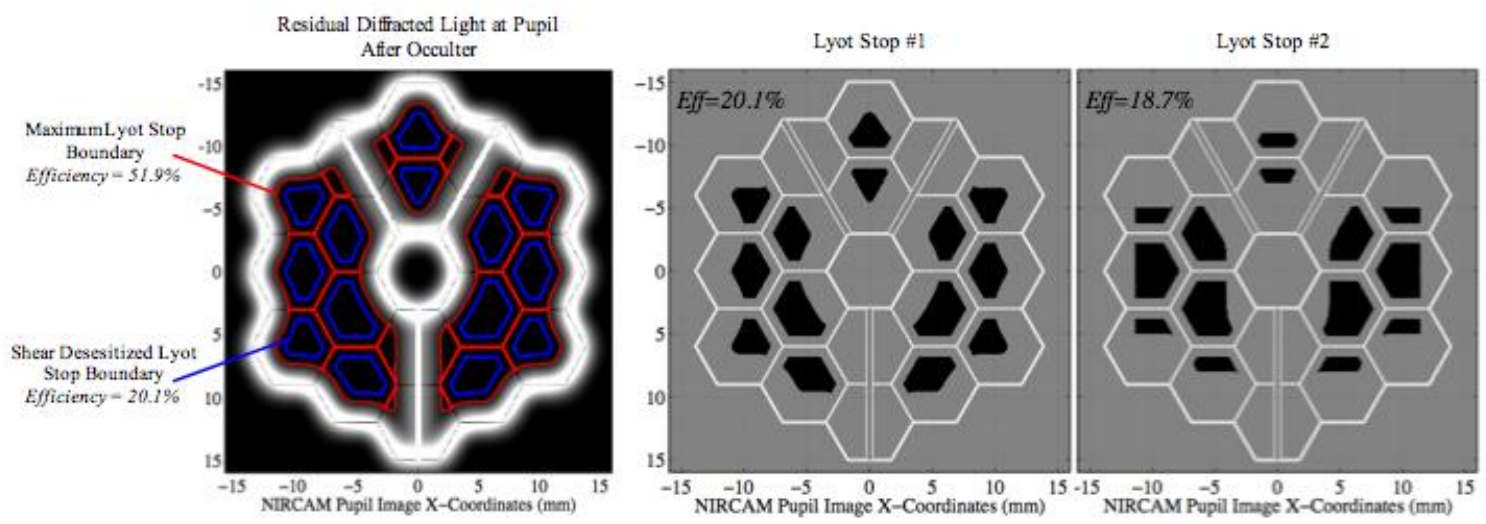

Figure 5. An example of the residual diffracted stellar light at the NIRCAM pupil is shown (left) along with the required Lyot stop designs (right) that are matched with the occulting spots shown in Fig 4. The Lyot stop designs are shown with an contour line overlay of the JWST pupil image. The openings in the Lyot stops are in black while the gray areas are masked out.

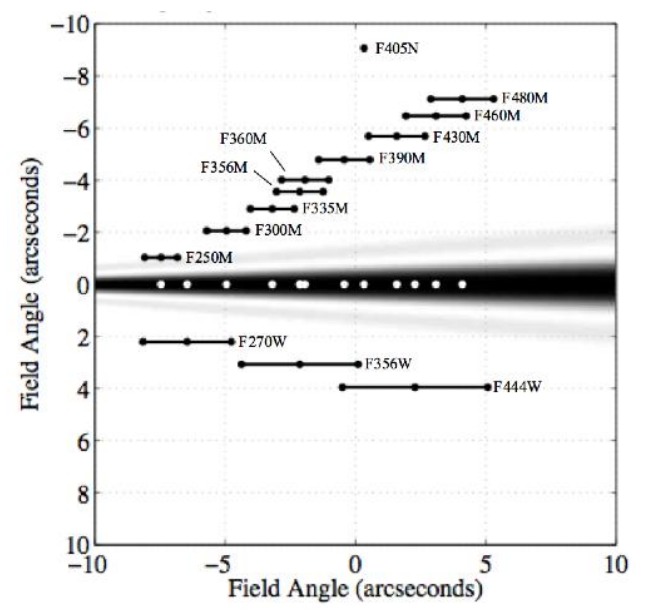

Figure 6. The pointing objectives that optimize the IWA per filter in the long-wave arm are shown. For a given stellar alignment, the coronagraph will work for all filters shorter than and including the one shown in the figure.

\section{HIGH CONTRAST IMAGING PERFORMANCE OF THE NIRCAM CORONGRAPH}

While the coronagraphs are well designed to eliminate the diffracted light from the JWST aperture, there is high level of scattered light caused by the optical aberrations. The wavefront error (WFE) in JWST will result from residual misalignments of the hexagonal segments and the secondary mirror as well as from the segment figure and surface quality that is achieved during polishing. In Fig. 7 we show an example realization of WFE that is consistent with the error budget for the telescope optics. We do not include the WFE allocated to NIRCAM in this analysis because the coronagraph suppresses the starlight before these errors are encountered. Also shown is the nominal imaging contrast that is obtainable without and with the 1.25 " radial-sombrero ${ }^{2}$ coronagraph using the F480M filter. Contrast is defined as the integrated scattered light in a diffraction-limited resolution spot, normalized by the coronagraph mask throughput, and divided by the light from the star that would be present without a coronagraph mask ${ }^{12}$.

In Fig. 8, we show the 3- $\sigma$ envelopes for the azimuthally averaged contrast for nominal imaging performance as well as the coronagraphic performance with three states of WFE. These states represent the expected operational telesope WFE $(111 \mathrm{~nm} \mathrm{rms})$ as well as with half the segment misalignments $(87 \mathrm{~nm} \mathrm{rms})$ and with only the segment fabrication errors $(31 \mathrm{~nm} \mathrm{rms})$. In this figure we also show the performance of hard-edged occulter with an equivalent IWA. 

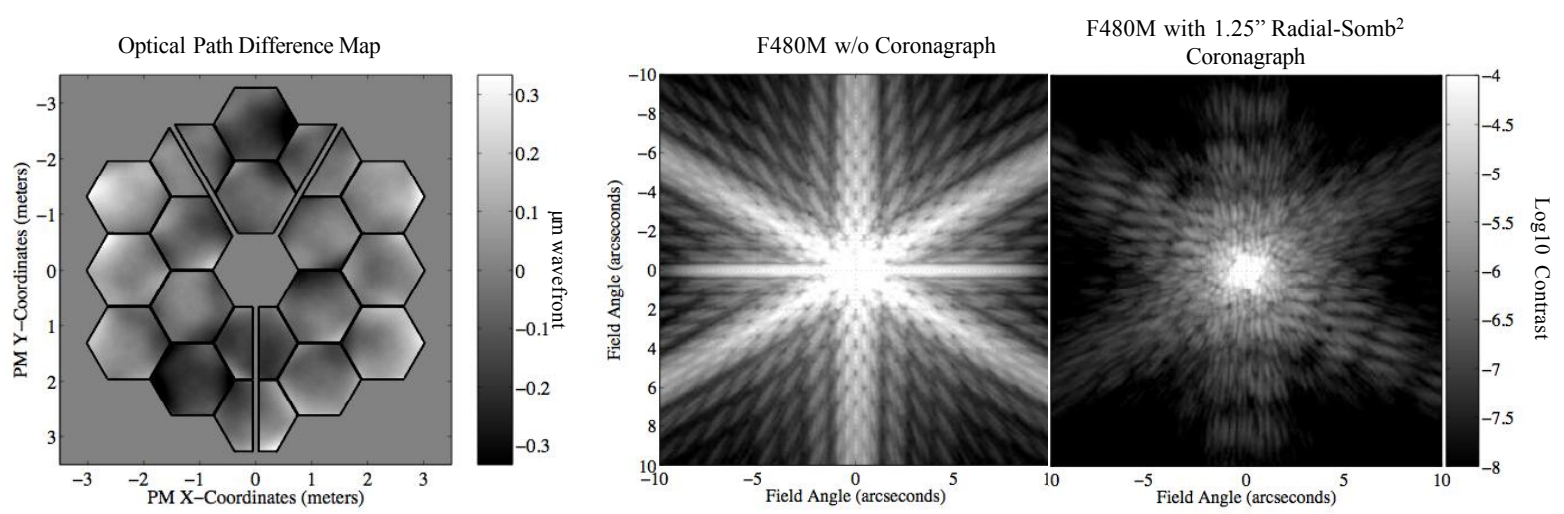

Figure 7: An example of the expected operational telescope wavefront error is shown (left) along with the imaging contrast one obtains without and with a coronagraph.
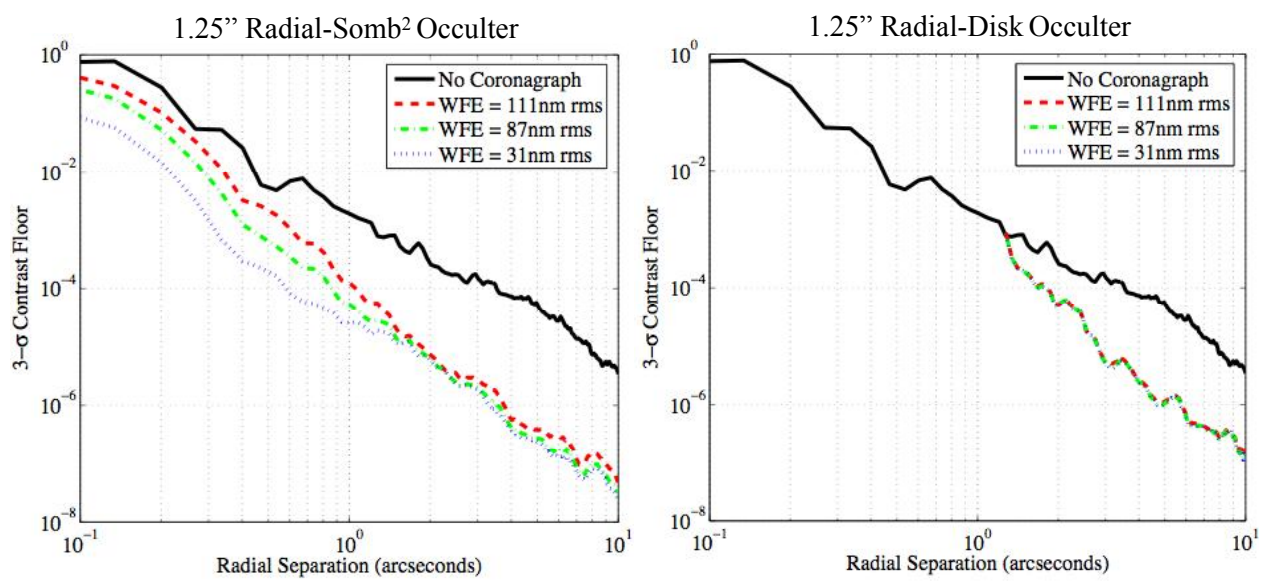

Figure 8. 3- $\sigma$ contrast envelopes to the azimuthally averaged contrast are shown for both the nominal (non-coronagraphic) imaging performance as well as for the coronagraphic imaging performance with three different states of WFE. The curves on the left represent the performance of the 1.25 " radial-sombrero ${ }^{2}$ occulter with the F480M filter. The curves on the right show the performance of a hard-edged radial-disk occulter.

Even in the presence of substantial WFE, using an apodized occulting spot facilitates a tighter IWA while reducing the residual starlight in the focal-plane by a factor of 10. Fig. 8 also reveals how the structure of the WFE affects the contrast floor. Most notably is lack of influence of segment alignment errors upon contrast at separations greater than $\sim 1.5$ ". The floor is dominated by the residual segment surface errors that will present stable speckle patterns that will be high amenable to calibration.

Ultimately, we are interested in detecting sources that are fainter than the residual scattered light cause by the WFE. Depending upon the stability level of the residual starlight speckles, there are many potential calibration/removal schemes. These include:

(1) Roll-and-subtract imaging: Subtract two images taken at difference roll angles about the target star.

(2) Calibration star subtraction: Subtract off speckle pattern acquired from a color-matched star that has no companion but is of own.

(3) Wavefront sensing based calibration: Recreate the speckle pattern to remove using an optical model derived from wavefront sensing information.

(4) Speckle deconvolution: Combine two or more images of the target star taken with different filters 
Although method (1) is straight forward, JWST will have limited capacity to roll about the target star. this method also requires that star maintains of alignment to the occulter, complicating the telescope operation. Nonetheless, roll-andsubtract will have some utility for companion source detection at small working angles.

Matching the color of the calibration star in method (2) may be problematic. Variation in the flux density of the reference star (as compared to the target) will limit the quality of the subsequent subtraction. If the suitable star can be found, this approach valuable to reducing speckle in order to reveal faint extended objects such as protoplanetary disks.

JWST will employ wavefront sensing schemes to periodically maintain the telescope alignment ${ }^{14}$. Instead of using a calibration star, an optical model that incorporates wavefront sensing information may create a speckle pattern within a desired optical passband. As in method (2), this method may also enable the observation of extended diffuse objects about the target star.

As with the JWST fine-guidance camera corongraph ${ }^{13}$, the array of filters in NIRCAM may well enable some speckle deconvolution approach. To illustrate the approach, we consider a basic two-color image subtraction scheme. Before combining the frames, we calibrate out the occulter transmission loss by

$$
\operatorname{img}_{n}^{\prime}(x, y)=i m g_{n}(x, y) \cdot \frac{T(x, y)}{|T(x, y)|^{2}+\varepsilon},
$$

where $\varepsilon$ is inversely related the imaging SNR and serves to limit noise amplification. To suppress the speckles while leaving any potential companions intact, we combine the images as

$$
\operatorname{diff}(x, y)=\operatorname{img}_{1}(x, y)-\left(\frac{\lambda_{1}}{\lambda_{2}}\right)^{2} \operatorname{img}_{2}\left(x \cdot \frac{\lambda_{2}}{\lambda_{1}}, y \cdot \frac{\lambda_{2}}{\lambda_{1}}\right),
$$

where $\lambda_{1}=3.56 \mu \mathrm{m}$ and $\lambda_{2}=3.90 \mu \mathrm{m}$. In Fig. 9, we show two images taken through the 1.25 " radial-sombrero ${ }^{2}$ occulter using the F356M and F390M filters after they have been calibrated by eqn. (4). The difference image computed by eqn. (5) is also shown. It nicely reveals the $1 \times 10^{-4}$ point source at a 0.75 " separation. Also, the $1.5 \times 10^{-5}$ sidelobes of the companion PSF are also quite visible. This simple technique suppressed the stellar speckles by a factor of 5-10.
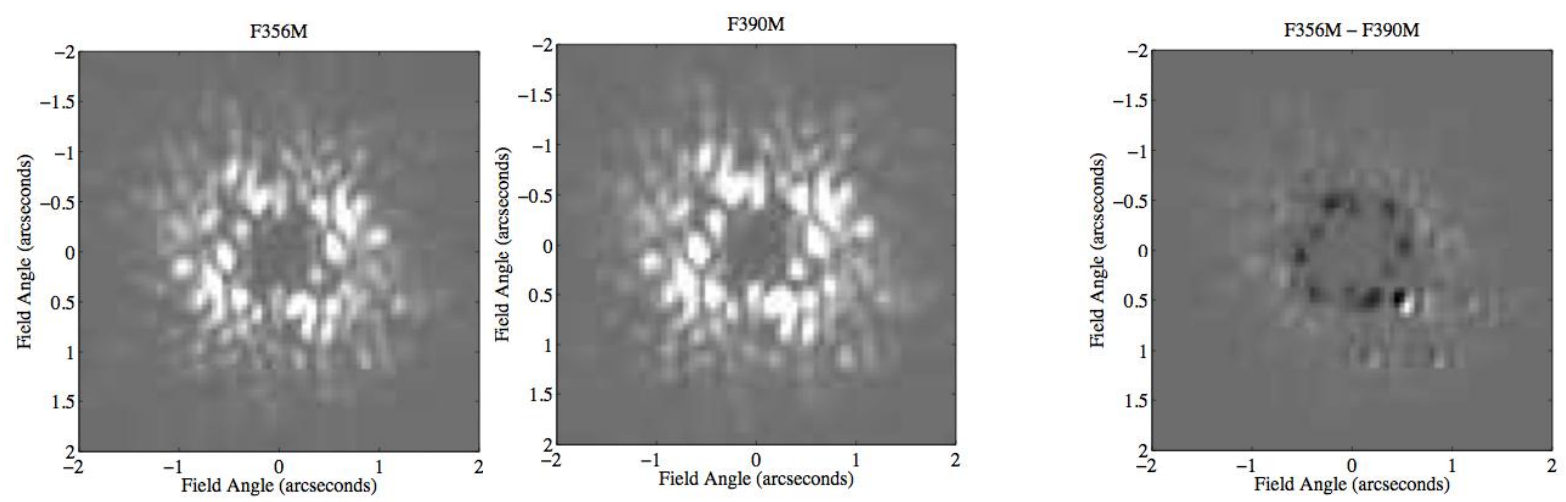

Figure 9. Example of two-color image subtraction is shown. The images on the left show the residual scattered starlight along with a $10^{-4}$ companion at 0.75 ". These images have been processed to normalize out the 1.25 " radial occulter transmission function. After the F390M is appropriately rescaled and resampled it is subtracted off from the F356M image to yield the image on the right. 


\section{CLOSING REMARKS}

JWST will be the premier facility for conducting coronagraphic searches for planets and protoplanetary disks in the 3$5 \mu \mathrm{m}$ range. As we have shown, the array of occulting spots in NIRCAM can provide a great range of operational flexibility to optimize the science return. While we have shown one method for speckle subtraction, there remain numerous other promising schemes that may offer significant advantages depending on the specific application. In particular, we plan to explore wavefront-sensing based schemes that enable better speckle subtraction in the presence of extended objects (debris disks, jets ect.). Aside from the baseline JWST focus-divers wavefront sensing scheme, we wish to examine the utility of a post-coronagraph wavefront sensing method ${ }^{15}$ that involves using the NIRCAM pupil imaging mode with the coronagraph aligned. We will also look at ways of employing more efficient Lyot stops. This would require using the actuated pickoff mirror in NIRCAM to better align the pupil image to the Lyot stops. More efficient Lyot stops would offer the ability to conduct deeper searches faint companions close to bright stars.

\section{ACKNOWLODGEMENTS}

The research described in this paper was carried out at the Jet Propulsion Laboratory, California Institute of Technology, under a contract with the National Aeronautics and Space Administration. It was supported by a contract from the NIRCAM instrument project at the University of Arizona.

\section{REFERENCES}

1. Rieke, M. et al., "NGST NIRCam Scientific Program and Design Concept," Proc. SPIE Vol. 4850 pp. $478-485$ (2003).

2. Burrows, A., Marley, M., Hubbard, W.B., Lunine, J.I., Guillot, T., Saumon, D., Freedman, R., Sudarsky, D., Sharp, C. 1997, ApJ, 491, 856.

3. Sudarsky, D., Burrows, A. and Pinto, P., “Albedo and Reflection Spectra of Extrasolar Giant Planets,” 2000, ApJ, v538.

4. Chauvin, G., Lagrange, A.-M., Dumas, C., Zuckerman, B., Mouillet, D., Song, I., Beuzit, J.-L., Lowrance, P., 2005, A\&A, 438, L25.

5. Burrows, A., Sudarsky, D., and Hubeny, I. 2004, ApJ, 609, 407.

6. Kalas, P. Graham, J. R., Clampin, M. 2005, Nature, 435, 1067

7. Ardila et al. (2005), ApJ., 627,986

8. Emery, J.P. and Brown, R.H. 2003, Icarus, 164, 104

9. Dekany, R. Stapelfeldt,K., Traub, W. Macintosh, B., Woolf, N., Colavita, M., Trauger, J., and Ftaclas, C. 2005, preprint.

10. Roberge, A. Weinberger, Alycia J., Malumuth, E. M., 2005, ApJ, 622, 171

11. Kuchner M. J., and Traub W. A., "A Coronagraph with a Band-Limited Mask for Finding Terrestrial Planets," ApJ 570, pp. 900-908 (2002).

12. Green J. J., and Shaklan S. B., "Optimizing Coronagraph Performance Designs to Minimize Their Contrast Sensitivity to LowOrder Optical Aberrations,” Proc. SPIE Vol. 5170, pp. 25-37 (2003).

13. Doyon, R. et al, "High-contrast imaging performance of the JWST fine guider sensor tunable filter coronagraph," Proc. SPIE Vol. 5487, (2004).

14. Green, J. J., et al, "Target selection and imaging requirements for JWST fine phasing," Proc. SPIE, vol 5487, (Glasgow 2004).

15. Green, J. J., et al, "Post-Coronagraph Wavefront Sensing Using Pupil Imaging and Phase Diversity," Opt. Soc. Am., SRS Meeting (Charlotte, NC) June 2005. 